\section{Estresse no trabalho e interrupção de atividades habituais, por problemas de saúde, no Estudo Pró-Saúde}

\author{
Job stress and interruption of routine activities due \\ to health problems, according to the Pró-Saúde \\ Study
}

${ }_{1}^{1}$ Escola Nacional de Saúde Pública Sergio Arouca, Fundação Oswaldo Cruz, Rio de Janeiro, Brasil. 2 Instituto de Medicina Social, Universidade do Estado do Rio de Janeiro, Rio de Janeiro, Brasil.

Correspondência L. E. T. Macedo Escola Nacional de Saúde Pública Sergio Arouca, Fundação Oswaldo Cruz. Rua Leopoldo Bulhões 1480, Rio de Janeiro, $R J$ 21041-210, Brasil. mced7@hotmail.com

\begin{abstract}
Health-related complaints have been associated with work problems more frequently than with any other aspect of life, including financial and family problems. This study focuses on the association between stress at work and interruption of routine activities due to health problems. The study adopted a cross-sectional approach and is part of a prospective study of 2,343 technical and administrative employees at a public university in the State of Rio de Janeiro, Brazil, known as the Pró-Saúde Study. Multivariate analyses were conducted using the generalized linear models and assuming a binomial distribution and log-link function. Men in jobs with heavy demands showed twice the prevalence of interruption of routine activities as compared to those not classified in this category (low demand; active; passive), after adjusting for age $(P R=2.06$; 95\%CI: 1.54-2.76). Among women, the outcome prevalence was $45 \%$ greater $(P R=$ 1.45; 95\%CI: 1.17-1.79).
\end{abstract}

Stress; Activities of Daily Living; Occupational Health
Luís Eduardo Teixeira de Macedo ${ }^{1}$

Dóra Chor ${ }^{1}$

Valeska Andreozzi 1

Eduardo Faerstein 2

Guilherme L. Werneck 2

Claudia S. Lopes 2

\section{Introdução}

Nos Estados Unidos, queixas relativas à saúde têm sido mais freqüentemente associadas a problemas no trabalho do que a qualquer outro aspecto da vida, incluindo problemas financeiros ou familiares. Naquele país, um quarto dos trabalhadores percebe sua ocupação como o primeiro agente causador de estresse em suas vidas (National Institute for Ocupational Safety and Health. http://www.cdc.gov/niosh/topics/stresswk. acessado em Out/2004).

A reação do organismo ao estresse visa sua proteção, preparando-o para o enfrentamento ou fuga da situação ameaçadora. Freqüência cardíaca, freqüência respiratória, concentração de glicose no sangue e quantidade de energia armazenada como gordura, sofrem alterações em um organismo sob estresse, devendo retornar aos limites da normalidade quando cessarem os estímulos. Segundo McEwen \& Lasley 1, essas são reações "alostáticas", aquelas que, visando à manutenção do equilíbrio interno do organismo, podem oscilar dentro de uma faixa de normalidade mais ampla do que a das reações homeostáticas (temperatura corporal, por exemplo). As reações "alostáticas" cumprem seus propósitos em curto prazo, mas, ao se perpetuarem, podem causar desgaste e doença caracterizando o que tem sido denominado de "sobrecarga alostática".

A teoria da sobrecarga alostática procura compreender melhor os mecanismos cerebrais 
envolvidos nesse processo, desde a percepção da situação estressora até a ativação do eixo hipotálamo-pituitário-adrenal e a resposta do restante do organismo aos estímulos nervosos e hormonais. Ela dá seqüência aos relatos de Hans Selye (1936, apud McEwen \& Lasley ${ }^{1}$ ) que, desde 1936, já identificava a resposta ao estresse como um fenômeno endócrino (Síndrome de Adaptação Geral), capaz de provocar doenças diversas, inclusive mentais.

Em 1979, Karasek 2 concebeu um modelo de avaliação do estresse percebido no ambiente de trabalho, composto de duas dimensões: demandas psicológicas e controle. Demandas psicológicas se referem à sobrecarga de trabalho, ao grau de dificuldade para a execução de tarefas, ao tempo disponível e ao ritmo empreendido para tal, assim como à existência de ordens contraditórias ou discordantes. Controle se refere à autonomia sobre as próprias tarefas e à motivação advinda da possibilidade de ser criativo e de usar, desenvolver e adquirir novas habilidades.

Baseando-se na percepção do trabalhador acerca do nível de demanda e controle, Karasek elaborou uma escala de medida do estresse no trabalho, com 49 questões 3 , posteriormente reduzida para 17 itens por Theorell et al. 4 . Por meio dessas escalas é possível classificar as atividades laborais em quatro situações: alta demanda e baixo controle (alta exigência); baixa demanda e alto controle (baixa exigência); alta demanda e alto controle (trabalhos ativos); e baixa demanda e baixo controle (trabalhos passivos) ${ }^{5}$. Ainda segundo esse modelo, atividades de alta exigência representariam o maior risco para os desfechos de saúde.

A associação entre estresse no trabalho e desfechos relacionados às doenças cardiovasculares está entre as mais freqüentemente investigadas 6,7 . Outros desfechos investigados foram a saúde autopercebida 8 , a depressão ${ }^{9}$ e o absenteísmo 10,11. No Brasil, Araújo et al. 12 utilizaram a escala completa ${ }^{3}$ para investigar a relação entre estresse no trabalho e a ocorrência de distúrbios psíquicos menores (não-psicóticos) entre trabalhadoras de enfermagem em Salvador, Bahia. Neste estudo, os distúrbios psíquicos menores associaram-se positivamente com as demandas psicológicas e negativamente com o controle sobre o trabalho.

As associações entre estresse no trabalho e desfechos relacionados à vitalidade e ao desempenho de atividades habituais - incluindo as laborais - têm sido pouco estudadas, e não temos conhecimento de investigações dessa natureza no Brasil. Cheng et al. 13, analisando dados do Nurses' Health Study (Estados Unidos), encontraram associação positiva com o declínio da capa- cidade de executar diversas atividades e tarefas cotidianas que requerem esforço físico. Lerner et al. 14 estimaram maior vitalidade e melhor funcionamento físico e social nos grupos classificados como desempenhando atividades laborais com menores níveis de estresse (os ativos e os de baixa exigência), quando comparados com o grupo com tarefas de alta exigência.

O estresse no trabalho tem sido apontado como um dos possíveis mecanismos de explicação para a relação entre desigualdades sociais e o risco de adoecer 15. Este trabalho pretende colaborar para o aprofundamento da compreensão desses mecanismos, ao investigar a hipótese de que indivíduos que desempenham tarefas classificadas como de alta exigência devido ao estresse no trabalho apresentam maior freqüência de interrupção das atividades habituais, tais como trabalho, estudo, lazer ou tarefas domésticas. Ao investigar esse desfecho buscamos captar o impacto do estresse na capacidade funcional do ponto de vista físico, social e emocional, cuja diminuição pode preceder, seguir ou ser independente da existência de doença percebida ou diagnosticada.

\section{Métodos}

\section{Participantes}

Esta investigação, do tipo transversal, se insere em um estudo prospectivo dos funcionários técnico-administrativos de uma universidade no Estado do Rio de Janeiro - Estudo Pró-Saúde. Duas fases de coleta de dados foram realizadas (1999 e 2001). Participaram de ambas as coletas 3.253 (78\%) funcionários dentre 4.160 funcionários elegíveis, excluindo-se apenas aqueles aposentados ou cedidos a outras instituições.

As análises apresentadas neste artigo são baseadas em 2.343 participantes de ambas as coletas de dados, tendo sido excluídos aqueles com dados faltantes em qualquer dos itens da escala de medida de estresse no trabalho, ou na pergunta a respeito de interrupção de atividades habituais por motivo de doença, ou em qualquer uma das covariáveis (renda, escolaridade, ocupação, trabalho em regime de plantão noturno, relato de diagnóstico de doença crônica, consumo de bebidas alcoólicas, escala de medida de transtornos mentais comuns).

\section{Medidas}

Nas duas fases de coleta de dados os participantes responderam a questionários autopreenchíveis, e tiveram peso e altura (fases 1 e 2), pressão 
arterial e circunferência da cintura (fase 2) aferidos.

Tanto as questões referentes à exposição quanto a pergunta referente ao desfecho aqui avaliado foram incluídas apenas no questionário da fase 2 de coleta de dados. A exposição - estresse no trabalho - foi avaliada através da versão sueca reduzida da escala completa. Esta escala foi traduzida e adaptada para o português no âmbito do Estudo Pró-Saúde 16 e contém cinco questões para avaliar a demanda; seis, para o controle, e outras seis que abordam o apoio social (não incluído nessa investigação). Quatro opções de resposta para as dimensões de demanda e de controle foram apresentadas utilizando-se uma escala tipo Likert, variando de "freqüentemente" até "nunca/quase nunca".

Quanto ao desfecho, foi avaliado através da pergunta: "Nas últimas duas semanas, você ficou impedido(a) de realizar alguma de suas atividades habituais (por exemplo, trabalho, estudo, lazer ou tarefas domésticas) por algum problema de saúde que você teve ou tem?", que poderia ser respondida afirmativa ou negativamente. No caso afirmativo, perguntou-se qual foi o problema de saúde responsável pelo impedimento, se o respondente procurou assistência médica visando tratamento, e também sobre a duração (número de dias) da interrupção das atividades habituais. Essa pergunta foi sugerida em publicação da Organização Mundial da Saúde 17 em 1996, como uma das formas de avaliação de incapacidades temporárias. No Brasil, constou da Pesquisa Nacional por Amostras de Domicílios (PNAD) saúde em 1998 e foi utilizada em alguns artigos nacionais 18,19 .

Um estudo de confiabilidade teste-reteste do questionário aplicado na fase 2 foi realizado com 94 funcionários contratados, mas não efetivos da universidade. De acordo com os resultados desse estudo, a confiabilidade da pergunta sobre interrupção de atividades habituais foi estimada em 0,73 (IC95\%: 0,71-0,75) (coeficiente kappa). A confiabilidade das dimensões do estresse no trabalho foi avaliada através dos coeficientes de correlação intraclasse, estimando-se 0,88 para demanda (IC95\%: 0,82-0,92) e 0,87 para controle (IC95\%: 0,80-0,91). Quanto à consistência interna das escalas, os coeficientes Alpha de Cronbach foram 0,72 para demanda e 0,63 para controle 16 .

Outras características analisadas foram: idade; escolaridade (fundamental: até $1^{\circ}$ grau completo; médio: $2^{\circ}$ grau completo; e universitário); renda mensal domiciliar per capita em salários mínimos (tercis); classe ocupacional, de acordo com a classificação de Erikson \& Goldthorpe 20, (profissionais, trabalhadores com rotina manual e trabalhadores com rotina não manual); traba- lho em regime de plantão noturno (categorizado em "sim" ou "não", e período de tempo acumulado: nunca; menos de 1 ano; 1 a 5 anos e mais de 5 anos); presença de doença crônica ("sim” ou "não" de acordo com o auto-relato de diagnóstico médico ao longo da vida de uma ou mais das seguintes condições: hipertensão arterial; diabetes mellitus; doença pulmonar obstrutiva crônica e afecções reumáticas crônicas); consumo de bebidas alcoólicas (periodicidade e quantidade consumida) nas últimas duas semanas: nenhum consumo; baixo consumo (1-7 doses em um único dia; ou 1-4 doses em 2 a 5 dias; ou 1 dose em 6 a 9 dias); consumo moderado (8 ou mais doses em um único dia; ou 5-10 doses em 2 a 5 dias; ou 2 - 7 doses em 6 a 9 dias; ou apenas 1 dose em 10 ou mais dias); e alto consumo (mais de 10 doses em 2 a 5 dias; ou 8 ou mais doses em 6 a 9 dias; ou 2 ou mais doses em 10 a 13 dias; ou 2 ou mais doses todos os dias). A classificação de presença ou ausência de transtornos mentais comuns foi realizada por meio da análise das respostas à versão abreviada do General Health Questionnaire, em português, considerando-se positivos aqueles que responderam afirmativamente a pelo menos 3 das 12 perguntas que compõem o instrumento 21 .

\section{Análise de dados}

O estresse no trabalho foi analisado de acordo com os quadrantes propostos por Karasek (http://www.uml.edu/Dept/WE/people/ faculty/karasek.htm, acessado em Jan/2005), definidos segundo as medianas das escalas de demanda e controle. Assim, o grupo com baixo controle foi aquele com escore menor do que 17; alto controle: maior ou igual a 17; baixa demanda: menor ou igual a 14; e alta demanda: maior do que 14 .

Foram realizadas análises univariadas, bivariadas e estratificadas para fins de verificação de associação entre alta exigência devido ao estresse no trabalho e interrupção das atividades habituais com cada uma das covariáveis estudadas.

Utilizamos o teste qui quadrado para verificar se as associações encontradas apresentavam significância estatística ( $\mathrm{p}<0,10$ ), valendo-nos deste critério para a seleção das possíveis variáveis de confusão. Aquelas associadas tanto à exposição quanto ao desfecho foram testadas nos modelos multivariados.

Participantes com atividades classificadas como de alta exigência foram comparados com aqueles alocados em todas as outras categorias de estresse no trabalho (passiva, ativa e de baixa exigência). A medida de associação utilizada foi a razão de prevalência, estimada separada- 
mente para homens e mulheres. Dado o caráter dicotômico da variável resposta, procederam-se as análises multivariadas valendo-se dos modelos lineares generalizados e assumindo-se uma distribuição binomial e função de ligação logarítmica ${ }^{22}$. Inicialmente, as razões de prevalência e seus respectivos intervalos de $95 \%$ de confiança foram estimados em um modelo que continha apenas as variáveis de exposição e de desfecho. Posteriormente, acrescentamos, uma de cada vez, a idade, os indicadores de posição sócio-econômica (escolaridade, renda e ocupação) e, em seguida, trabalho em plantões noturnos, consumo de álcool, relato de diagnóstico de doença crônica e classificação de transtornos mentais comuns. No modelo final, foi mantida a covariável que alterou a associação entre estresse no trabalho e interrupção das atividades habituais em pelo menos $10 \%$, sendo idade forçada no modelo. O programa de domínio público utilizado foi o R versão 2.4.1 (The R Foundation for Statistical Computing. http:/ / www.R-project. org).

\section{Aspectos éticos:}

A pesquisa não envolveu riscos físicos, sociais ou legais aos participantes, embora dados a respeito de questões consideradas delicadas tenham sido coletados (consumo de bebida alcoólica, uso de medicamentos, diagnóstico de doenças, entre outros). Os dados foram analisados somente de forma agregada, sem individualizações. A participação na pesquisa foi voluntária, tendo sido esclarecido aos funcionários que nenhum tipo de penalidade seria aplicada aos que não desejassem participar. Após esclarecimentos sobre a pesquisa, foi solicitada a assinatura de Consentimento Informado para Participação em Pesquisa Científica.

\section{Resultados}

Trata-se de uma população jovem - aproximadamente $70 \%$ tinha menos que 45 anos de idade, sendo as mulheres um pouco mais velhas do que os homens. As mulheres (56\%) compunham a maior parte do grupo estudado. Comparadas aos homens, apresentaram maior renda familiar per capita e melhor nível de escolaridade. A ocupação de cerca de dois terços dos participantes foi classificada como não manual de rotina (Tabela 1). A prevalência de alta exigência foi maior entre as mulheres (15,9\%) comparadas aos homens $(11,6 \% ; p=0,003)$ assim como a prevalência da interrupção das atividades habituais - 26,3\% e $18,2 \%$, respectivamente $(\mathrm{p}<0,001)$.
A prevalência de alta exigência não variou significativamente com a idade (Tabela 2). Entre as mulheres (mas não entre os homens), variou de forma inversa com a escolaridade: $21,9 \%$ entre aquelas que apresentavam apenas o nível fundamental de ensino, 18,9\% entre aquelas com nível médio, e 12,4\% entre as de nível universitário ( $\mathrm{p}<$ 0,001 ). De maneira semelhante, quanto menor a renda mensal domiciliar per capita, maior a prevalência de atividades de alta exigência apenas entre as mulheres $(p=0,001)$. No caso da ocupação, essa tendência foi observada entre homens e mulheres: aqueles classificados como trabalhadores manuais apresentaram maior prevalência de alta exigência do que aqueles com ocupação de rotina não manual que, por sua vez, apresentaram maior prevalência da exposição do que os profissionais. Em relação aos transtornos mentais comuns, a prevalência de alta exigência foi significativamente maior entre os participantes classificados como positivos comparados aos negativos, tanto entre os homens $(17,4 \%$ vs. 9,4\%) $(\mathrm{p}<0,001)$ quanto entre as mulheres $(23,4 \% v s$. $11,3 \%$; $<<0,001$ ).

A prevalência de interrupção das atividades habituais não variou de forma importante entre as categorias de escolaridade, em ambos os sexos. Somente entre os homens variou de forma inversa com a renda domiciliar per capita: $21,4 \%$ entre aqueles que ganhavam até 3 salários mínimos; $16,7 \%$ entre os que ganhavam na faixa de 3 a 6 salários mínimos e 14,5\% entre os que ganhavam mais de 6 salários mínimos ( $p=0,05)$. Entre as mulheres, aquelas com atividades não manuais de rotina apresentaram a maior prevalência do desfecho, bem como observou-se gradiente positivo entre o tempo de trabalho em regime de plantão noturno e a prevalência do desfecho, não significante estatisticamente $(p=0,11)$.

Em ambos os sexos, a prevalência de interrupção das atividades habituais foi significativamente maior entre aqueles classificados como positivos para os transtornos mentais comuns ( $p<0,001$ ), assim como entre os que relataram doença crônica, comparados aos que não as relataram (homens: $\mathrm{p}=0,10$; mulheres: $\mathrm{p}<0,001$ )

Os homens em atividades com alta exigência apresentaram prevalência da interrupção das atividades habituais duas vezes maior do que aqueles cujas atividades não foram classificadas nessa categoria (baixa exigência; ativo; passivo) ajustando-se por idade (Tabela 3). Entre as mulheres, a prevalência do desfecho foi $45 \%$ maior. As razões de prevalência variaram pouco (menos que $4 \%$ ) ao incluirmos as outras possíveis variáveis de confusão: ocupação, renda e doença crônica, no caso dos homens; escolaridade, renda, ocupação, plantão noturno e doenças 
Características sócio-demográficas, estresse no trabalho e interrupção das atividades habituais. Estudo Pró-Saúde, 2001.

\begin{tabular}{|c|c|c|c|c|c|c|}
\hline & \multicolumn{2}{|c|}{ Homens } & \multicolumn{2}{|c|}{ Mulheres } & \multicolumn{2}{|c|}{ Total } \\
\hline & $\mathbf{n}$ & $\%$ & n & $\%$ & $\mathbf{N}$ & $\%$ \\
\hline Sexo & 1.030 & 44,0 & 1.313 & 56,0 & 2.343 & \\
\hline \multicolumn{7}{|l|}{ Idade (anos) } \\
\hline$<35$ & 278 & 27,0 & 255 & 19,4 & 533 & 22,7 \\
\hline $35-44$ & 463 & 45,0 & 618 & 47,1 & 1.081 & 46,2 \\
\hline $45-54$ & 232 & 22,5 & 356 & 27,1 & 588 & 25,1 \\
\hline$>54$ & 57 & 5,5 & 84 & 6,4 & 141 & 6,0 \\
\hline \multicolumn{7}{|l|}{ Escolaridade } \\
\hline Fundamental & 218 & 21,1 & 187 & 14,2 & 405 & 17,2 \\
\hline Médio & 388 & 37,7 & 438 & 33,4 & 826 & 35,3 \\
\hline Universitário & 424 & 41,2 & 688 & 52,4 & 1112 & 47,5 \\
\hline \multicolumn{7}{|c|}{ Renda domiciliar per capita } \\
\hline \multicolumn{7}{|c|}{ (salários mínimos) } \\
\hline Até 3 & 435 & 42,2 & 399 & 30,4 & 834 & 35,6 \\
\hline $3-6$ & 353 & 34,3 & 495 & 37,7 & 848 & 36,2 \\
\hline$>6$ & 242 & 23,5 & 419 & 31,9 & 661 & 28,2 \\
\hline \multicolumn{7}{|l|}{ Ocupação } \\
\hline Profissionais & 283 & 27,5 & 359 & 27,3 & 642 & 27,4 \\
\hline Rotina não manual & 607 & 58,9 & 908 & 69,2 & 1.515 & 64,7 \\
\hline Rotina manual & 140 & 13,6 & 46 & 3,5 & 186 & 7,9 \\
\hline \multicolumn{7}{|l|}{ Estresse no trabalho } \\
\hline Alta exigência & 119 & 11,6 & 209 & 15,9 & 328 & 14,0 \\
\hline Baixa exigência & 304 & 29,5 & 320 & 24,4 & 624 & 26,6 \\
\hline Ativo & 225 & 21,8 & 338 & 25,7 & 563 & 24,0 \\
\hline Passivo & 382 & 37,1 & 446 & 34,0 & 828 & 35,4 \\
\hline \multicolumn{7}{|c|}{ Interrupção das atividades habituais } \\
\hline Sim & 187 & 18,2 & 345 & 26,3 & 532 & 22,7 \\
\hline Não & 843 & 81,8 & 968 & 73,7 & 1.811 & 77,3 \\
\hline
\end{tabular}

crônicas, no caso das mulheres. Ao ajustarmos pela presença de transtornos mentais comuns, houve importante redução das razões de prevalência. Mesmo assim, os homens com atividades de alta exigência apresentaram prevalência $70 \%$ maior de interrupção de suas atividades habituais (IC 95\%: 1,28-2,25), enquanto entre as mulheres essa prevalência foi $23 \%$ maior (IC 95\%: 1-2,25).

Não se observaram diferenças estatisticamente significantes $(p=0,67)$ na prevalência de alta exigência entre aqueles que procuraram e os que não procuraram assistência médica em função da interrupção das atividades habituais, nem tampouco entre os participantes que interromperam atividades habituais por mais de sete dias comparados com aqueles que as interromperam por menor período de tempo $(p=0,73)$ (dados não apresentados).

\section{Discussão}

Nesse estudo, tarefas classificadas como de alta exigência associaram-se a risco 1,5 a 2 vezes maior de interrupção das atividades habituais por motivo de doença. Após ajuste por transtornos mentais comuns, o risco foi $69 \%$ maior entre homens, e $23 \%$ maior entre as mulheres.

A fim de identificar estudos a respeito da associação investigada, realizamos revisão não sistemática da literatura no Index Medicus da Biblioteca Nacional Americana de Medicina (MEDLINE) e na Scientific Electronic Library Online (SciELO), além de buscar referências em livros especializados e em conversas com especialistas. Uma vez que não foi possível encontrar nenhuma investigação sobre a associação em estudo, comparamos nossos resultados com as pesquisas cujos desfechos mais se aproximaram daquele analisado nesse trabalho. 
Prevalência de estresse no trabalho e de interrupção das atividades habituais devido a problemas de saúde, por sexo e covariáveis. Estudo Pró-Saúde, 2001.

\begin{tabular}{|c|c|c|c|c|c|c|c|c|c|c|}
\hline & \multicolumn{5}{|c|}{ Estresse no trabalho } & \multicolumn{5}{|c|}{ Interrupção das atividades habituais } \\
\hline & \multicolumn{2}{|c|}{ Homens } & \multicolumn{2}{|c|}{ Mulheres } & \multirow{2}{*}{$\begin{array}{c}\text { Total } \\
\%\end{array}$} & \multicolumn{2}{|c|}{ Homens } & \multicolumn{2}{|c|}{ Mulheres } & \multirow{2}{*}{$\begin{array}{c}\text { Total } \\
\%\end{array}$} \\
\hline & $\mathrm{n}$ & $\%$ & $\mathbf{n}$ & $\%$ & & $\mathbf{n}$ & $\%$ & $\mathrm{n}$ & $\%$ & \\
\hline \multicolumn{11}{|l|}{ Idade (anos) } \\
\hline$<35$ & 35 & 12,6 & 39 & 15,3 & 13,9 & 53 & 19,1 & 75 & 19,4 & 19,1 \\
\hline $35-44$ & 47 & 10,2 & 98 & 15,9 & 13,4 & 90 & 19,4 & 152 & 24,6 & 22,4 \\
\hline $45-54$ & 29 & 12,5 & 53 & 14,9 & 13,9 & 39 & 16,8 & 96 & 27,0 & 23,0 \\
\hline$>54$ & 8 & 14,0 & 19 & 22,6 & 19,1 & 5 & 8,8 & 22 & 26,2 & 24,0 \\
\hline \multicolumn{11}{|l|}{ Escolaridade } \\
\hline Fundamental & 28 & 12,8 & 41 & 21,9 * & 17,0 & 39 & 17,9 & 50 & 26,7 & 22,0 \\
\hline Médio & 48 & 12,4 & 83 & 18,9 & 15,9 & 73 & 18,8 & 132 & 30,1 & 24,8 \\
\hline Universitário & 43 & 10,1 & 85 & 12,4 & 11,5 & 75 & 17,7 & 163 & 23,7 & 21,4 \\
\hline \multirow{2}{*}{\multicolumn{11}{|c|}{$\begin{array}{l}\text { Renda domiciliar per capita } \\
\text { (em salários mínimos) }\end{array}$}} \\
\hline & & & & & & & & & & \\
\hline Até 3 & 56 & 12,9 & 94 & 23,6 * & 18,0 & 93 & 21,4 & 110 & 27,6 & 24,3 \\
\hline $3-6$ & 40 & 11,3 & 75 & 15,2 & 13,6 & 59 & 16,7 & 138 & 27,9 & 23,2 \\
\hline$>6$ & 23 & 9,5 & 40 & 9,5 & 9,5 & 35 & 14,5 & 97 & 23,2 & 20,0 \\
\hline \multicolumn{11}{|l|}{ Ocupação } \\
\hline Profissional & 14 & 4,9 ** & 35 & 9,7 *夫 & 7,6 & 46 & 16,3 & 70 & 19,5 ** & 18,1 \\
\hline Rotina não manual & 82 & 13,5 & 162 & 17,8 & 16,1 & 118 & 19,4 & 264 & 29,1 & 25,2 \\
\hline Rotina manual & 23 & 16,4 & 12 & 26,1 & 18,8 & 23 & 16,4 & 11 & 23,9 & 18,3 \\
\hline \multicolumn{11}{|l|}{ Plantão noturno (anos) } \\
\hline Nunca & 97 & 12,3 & 143 & 16,0 & 14,2 & 139 & 7,6 & 219 & 24,4 & 21,2 \\
\hline$<1$ & 4 & 10,8 & 10 & 14,5 & 13,2 & 9 & 24,3 & 18 & 26,1 & 25,5 \\
\hline 1 a 5 & 6 & 8,1 & 29 & 20,1 & 16,1 & 15 & 20,3 & 42 & 29,2 & 26,1 \\
\hline$>5$ & 12 & 9,4 & 27 & 13,2 & 11,7 & 24 & 18,8 & 66 & 32,4 & 27,1 \\
\hline \multicolumn{11}{|l|}{ Doença crônica } \\
\hline $\operatorname{Sim}$ & 50 & 13,7 & 91 & 17,1 & 15,7 & 76 & 20,8 & 174 & 32,6 ** & 27,8 \\
\hline Não & 69 & 10,4 & 118 & 15,1 & 13,0 & 111 & 16,7 & 171 & 21,9 & 19,5 \\
\hline \multicolumn{11}{|l|}{ Consumo de álcool } \\
\hline Nenhum & 48 & 11,7 & 128 & 16,5 & 14,8 & 73 & 17,8 & 207 & 26,6 & 23,6 \\
\hline Baixo & 37 & 10,2 & 65 & 15,6 & 13,1 & 62 & 17,0 & 109 & 26,2 & 21,9 \\
\hline Moderado & 26 & 14,9 & 15 & 16,0 & 15,2 & 35 & 20,0 & 20 & 21,3 & 20,4 \\
\hline Alto & 8 & 9,8 & 1 & 3,8 & 8,3 & 17 & 20,7 & 9 & 34,6 & 24,1 \\
\hline \multicolumn{11}{|c|}{ Transtornos mentais comuns } \\
\hline Sim & 49 & 17,4 ** & 17 & 23,4 ** & 21,3 & 97 & 34,4 ** & 197 & 39,5 ** & 37,6 \\
\hline Não & 70 & 9,4 & 92 & 11,3 & 10,4 & 90 & 12,0 & 148 & 18,2 & 15,2 \\
\hline
\end{tabular}

* $p<0,05$, para o teste de tendência linear;

** $p<0,05$.

De acordo com os resultados de diversas investigações 14,15,23,24, o estresse no trabalho e suas dimensões, demanda e controle, estão associados a diversos desfechos que representam interrupção de atividades habituais. No Estudo Whitehall II 25, de funcionários públicos ingleses, o risco de absenteísmo por motivo de saúde foi $50 \%$ menor nos indivíduos classificados como exercendo atividades com altos níveis de controle no trabalho, quando comparados aos fun- cionários com baixo controle $(\mathrm{RR}=0,50$; $\mathrm{IC} 95 \%$ : $0,47-0,54)$. Na mesma população, Stansfield et al. 15 relataram a existência de associações entre as dimensões do estresse no trabalho e diversas condições que devem ser levadas em consideração ao se investigar o risco de absenteísmo e interrupção de outras atividades habituais. Por exemplo, comparados aos participantes com altos níveis de controle no trabalho, aqueles com baixo controle apresentaram maior risco de 
Associação entre estresse no trabalho e interrupção de atividades habituais devido a problemas de saúde. Estudo Pró-Saúde 2001

\begin{tabular}{|c|c|c|}
\hline Modelos & Razão de prevalência * & IC95\% \\
\hline \multicolumn{3}{|l|}{ Homens } \\
\hline Modelo 1: estresse no trabalho & 2,08 & $1,55-2,79$ \\
\hline Modelo 2: estresse no trabalho + idade & 2,06 & $1,54-2,76$ \\
\hline Modelo 3: estresse no trabalho + idade + ocupação & 2,05 & $1,52-2,77$ \\
\hline Modelo 4: estresse no trabalho + idade + renda & 1,98 & $1,47-2,66$ \\
\hline Modelo 5: estresse no trabalho + idade + doença crônica & 2,02 & $1,51-2,71$ \\
\hline Modelo 6: estresse no trabalho + idade + transtornos mentais comuns & 1,70 & $1,28-2,25$ \\
\hline \multicolumn{3}{|l|}{ Mulheres } \\
\hline Modelo 1: estresse no trabalho & 1,44 & $1,17-1,78$ \\
\hline Modelo 2: estresse no trabalho + idade & 1,45 & $1,17-1,79$ \\
\hline Modelo 3: estresse no trabalho + idade + escolaridade & 1,41 & $1,14-1,74$ \\
\hline Modelo 4: estresse no trabalho + idade + renda & 1,42 & $1,15-1,76$ \\
\hline Modelo 5: estresse no trabalho + idade + ocupação & 1,40 & $1,14-1,73$ \\
\hline Modelo 6: estresse no trabalho + idade + plantão noturno & 1,47 & $1,19-1,81$ \\
\hline Modelo 7: estresse no trabalho + idade + doença crônica & 1,44 & $1,17-1,76$ \\
\hline Modelo 8: estresse no trabalho + idade + transtornos mentais comuns & 1,23 & $1,00-1,51$ \\
\hline
\end{tabular}

* Participantes com atividades classificadas como de alta exigência, comparados com aqueles em todas as outras categorias de estresse no trabalho (baixa exigência; ativo; passivo).

dependência alcoólica, especialmente entre as mulheres (OR = 1,46; IC95\%: 0,9-2,4); altos níveis de demanda dobraram o risco de transtornos mentais comuns em ambos os sexos $(\mathrm{OR}=2,02$; IC95\%:1,5-2,6) e, entre as mulheres, elevaram o risco de declínio da capacidade funcional social (OR = 1,75; IC 95\%:1,3-2,4).

Cheng et al. 23, avaliando uma coorte de enfermeiras americanas, encontraram associação positiva entre estresse no trabalho e declínio na capacidade física, com intensidade comparável àquelas estimadas em relação ao tabagismo ou sedentarismo. Na mesma coorte, Lerner et al. 14 estimaram, para o grupo de baixa exigência, chance $80 \%$ maior de apresentar mais vitalidade e melhor funcionamento físico e $68 \%$ maior de apresentar melhor funcionamento social do que o grupo de alta exigência. Josephson et al. ${ }^{24}$, estudando a ocorrência de sintomas músculo-esqueléticos entre enfermeiros de um hospital na Suécia, estimaram taxas $70 \%$ mais altas no grupo exposto às altas exigências no trabalho quando comparado aos demais.

Além da ausência de investigações sobre a associação de interesse em nosso estudo, as várias formas com que diferentes autores vêm analisando as medidas de estresse no trabalho representam dificuldade adicional na comparação de resultados. Os escores de controle e de demanda permitem que essas dimensões sejam analisadas separadamente, como variáveis contínuas ou categóricas, ou ainda segundo sua relação - a razão demanda/controle. Além disso, conforme postula Karasek (http://www.uml.edu/Dept/WE/ people/faculty/karasek.htm, acessado em Jan/2005), as duas dimensões devem ser categorizadas conjuntamente, de acordo com os níveis de controle (alto e baixo) e os níveis de demanda (alta e baixa), dando origem aos quatro quadrantes. Optamos por trabalhar com os quadrantes por ser a alternativa que mais reflete a concepção dos autores da escala, além de ser uma forma de análise bastante utilizada, o que favorece comparações futuras. Quanto ao melhor ponto de corte dos escores de demanda e controle para constituição dos quadrantes, optamos pelo uso da mediana, a medida mais comumente utilizada $26,27,28$.

Apesar da importância do absenteísmo, que foi apontado como forte preditor de mortalidade e como marcador de morbidade mais sensível do que as medidas de saúde usualmente disponíveis 6 , uma das limitações desse estudo refere-se à pergunta que avaliou o desfecho (ver seção de métodos). Essa pergunta não discriminou qual das atividades habituais foi interrompida, o que limita a comparabilidade com os estudos sobre o absenteísmo, tema mais freqüentemente inves- 
tigado no âmbito da interrupção de atividades habituais. Na etapa de elaboração do questionário, essa limitação foi identificada, optando-se por manter essa formulação a fim de minimizar o risco de informações incorretas a respeito da ausência do trabalho, por se tratar de uma coorte de trabalhadores.

Um outro potencial problema que merece destaque foi a necessidade de exclusão de 910 participantes ( $28 \%$ ) das análises, em função de dados faltantes em uma ou mais variáveis. Quando comparamos o perfil sócio demográfico dos participantes excluídos com o da população estudada não se encontrou diferenças importantes em relação à distribuição etária e por sexo, nem em termos da freqüência de exposição a situações de alta exigência no trabalho ou da prevalência de interrupção das atividades habituais. Entretanto, os participantes excluídos apresentavam menor escolaridade o que poderia sugerir que os mecanismos que levaram a esta exclusão podem não ter sido completamente aleatórios, qual seja, que pode haver motivos distintos que determinem a não resposta. O impacto destas exclusões nos resultados do estudo não é de simples avaliação, mas acredita-se que não devam ter sido significativas, já que a freqüência de não resposta foi relativamente baixa considerando-se cada variável isoladamente, além do que não se observaram mudanças importantes das estimativas de razões de prevalência com a inclusão ou não das variáveis nos modelos multivariados.

Segundo McEwen \& Lasley ${ }^{1}$, existe plausibilidade na hipótese de que o estresse no trabalho seja direta ou indiretamente responsável por doenças ou sintomas inespecíficos, que causariam a interrupção de atividades diárias. De fato, é possível conceber que indivíduos que se percebem submetidos à sobrecarga de trabalho executando tarefas com alto grau de dificuldade, na presença de exigências contraditórias e tendo pouca autonomia sobre suas decisões e poucas oportunidades de desenvolver novas habilidades, sintam-se ou tornem-se menos saudáveis.

Os mecanismos mediadores entre o estresse e a interrupção das atividades habituais podem ser múltiplos. Os transtornos mentais comuns, por exemplo, que apresentaram forte associação com a exposição e com o desfecho, podem ser compreendidos como variável interveniente ao invés de variável de confusão, já que é possível considerá-los como parte do caminho causal entre o estresse no trabalho e a interrupção das atividades habituais. Ao contrário das outras variáveis de confusão, essa foi a única característica que reduziu fortemente a medida bruta de associação. Em função dessa possibilidade, não consideramos os resultados ajustados pelos transtornos mentais como indicativo da magnitude da associação, mas aqueles ajustados apenas pela idade.

Do ponto de vista da agenda de saúde pública, nossos resultados indicam a importância da pesquisa e de ações de saúde relativas ao estresse no trabalho, cujos efeitos ainda são pouco conhecidos no Brasil. Em um ambiente em constante e intensa modificação, é preciso conhecer melhor não só as tarefas que se apresentam, como também as potencialidades, dúvidas e expectativas do trabalhador, e repensar essa relação. 


\section{Resumo}

Queixas relativas à saúde têm sido mais freqüentemente associadas a problemas no trabalho do que a qualquer outro aspecto da vida, incluindo problemas financeiros ou familiares. Nesse estudo, investigamos a associação entre estresse no trabalho e a interrupção das atividades habituais por problemas de saúde. Esta investigação, do tipo transversal, se insere em um estudo prospectivo de 2.343 funcionários técnico-administrativos de uma universidade no Estado do Rio de Janeiro - Estudo Pró-Saúde. Procederam-se as análises multivariadas valendo-se dos modelos lineares generalizados e assumindo-se uma distribuição binomial e função de ligação logarítmica. Os homens em atividades com alta exigência apresentaram prevalência da interrupção das atividades habituais duas vezes maior do que aqueles cujas atividades não foram classificadas nessa categoria (baixa exigência; ativo; passivo), ajustando-se por idade ( $R P=2$,06; IC95\%: 1,54-2,76). Entre as mulheres, a prevalência do desfecho foi $45 \%$ maior (RP = 1,45; IC95\%: 1,17-1,79).

Estresse; Atividades Cotidianas; Saúde do Trabalhador

\section{Colaboradores}

L. E. T. Macedo efetuou os cálculos e preparou as Tabelas, discutiu os resultados e analisou-os junto com a equipe. Redigiu o corpo do artigo, tendo trabalhado nele até a sua forma final. D. Chor, uma das coordenadoras do Estudo Pró-Saúde, participou ativamente da discussão dos resultados, tendo colaborado na redação do artigo até a sua forma final. V. Andreozzi participou ativamente da análise dos dados e da preparação das tabelas. E. Faerstein, G. L. Werneck e C. S. Lopes, também coordenadores do Estudo Pró-Saúde, participaram ativamente da discussão dos resultados, e colaboraram na redação do artigo.

\section{Referências}

1. McEwen BS, Lasley E. Carga alostática: quando a proteção dá lugar ao dano. In: McEwen BS, Lasley E, organizadores. O fim do estresse como nós o conhecemos. Rio de Janeiro: Editora Nova Fronteira; 2003. p. 79-93.

2. Karasek RA. Job demands, job decision latitude, and mental strain: implications for job redesign. Adm Sci Q 1979; 24:285-307.

3. Karasek RA. Job content questionnaire and user's guide. Lowell: Departament of Work Environment, University of Massachusets; 1985.

4. Theorell T, Perski AA, Akerstedt T. Changes in job strain in relation to changes in fluctuations in physiological state. Scand J Work Environ Health 1988; 14:189-96.

5. Theorel T. Working conditions and health. In: Berkman L, Kawaki I, editor. Social epidemiology. New York: Oxford University Press; 2000. p. 95-117.

6. Kivimaki M, Leino-Arjas P, Luukkonen R, Riihimaki H, Vahtera J, Kirjonen J. Work stress and risk of cardiovascular morbidity: prospective cohort study of industrial employees. BMJ 2002; 325:857.
7. Kuper H, Marmot M. Job strain, job demands, decision latitude, and risk of coronary heart disease within the Whitehall II study. J Epidemiol Community Health 2003; 57:147-53.

8. Ibrahim AS, Scott FE, Cole DC, Shannon HS, Eyles J. Women's work, health and quality of life. New York: The Haworth Press Incorporation; 2001. p. 105-24.

9. Broadhead WE, Blazer DG, George LK, Tse CK. Depression, disability days, and days lost from work in a prospective epidemiologic survey. JAMA 1990; 264:2524-8.

10. Peter R, Siegrist J. Chronic work stress, sickness absence, and hypertension in middle managers: general or specific sociological explanations? Soc Sci Med 1997; 45:1111-20.

11. Kivimaki M, Head J, Ferrie JE, Shipley MJ, Vahtera J, Marmot MG. Sickness absence as a global measure of health: evidence from mortality in the Whitehall II prospective cohort study. BMJ 2003; 327:364-9. 
12. Araújo TM, Aquino E, Menezes G, Santos CO Aguiar L. Aspectos psicossociais do trabalho e distúrbios psíquicos entre trabalhadores de enfermagem. Rev Saúde Pública 2003; 37:424-33.

13. Cheng Y, Kawachi I, Coakley EH, Scwartz J, Colditz G. Association between psycosocial work characteristics and health functioning in American women: prospective study. BMJ 2000; 320:1432-6.

14. Lerner DJ, Levine S, Malspeis S, D'Agostino RB. Job strain and health-related quality of life in a national sample. Am J Public Health 1994; 84:1580-5.

15. Stansfeld S, Head J, Marmot M. Work related factors and ill health. The Whitehall II Study. Health \& Safety Executive 2000. http://www.hse.gov.uk/ research/crr_pdf/2000/crr00266.pdf (acessado em Nov/2004).

16. Alves MGM, Chor D, Faerstein E, Lopes CS, Werneck GL. Versão resumida da "Job Stress scale": adaptação para o português. Rev Saúde Pública 2004; 38:164-71.

17. Bruin A, Picavet HSJ, Nossikov A. Health Interview Surveys: towards international harmonization of methods and instruments. Geneva: World Health Organization; 1996. (Série Européia de Publicações, 58).

18. Travassos C, Viacava F, Pinheiro R, Brito A. Utilização dos serviços de saúde no Brasil: gênero, características familiares e condição social. Rev Panam Salud Pública 2002; 11:365-73.

19. Lima-Costa MF, Barreto SM, Giatti L. Condições de saúde, capacidade funcional, uso de serviços de saúde e gastos com medicamentos da população idosa brasileira: um estudo descritivo baseado na Pesquisa Nacional por Amostra de Domicílios. Cad Saúde Pública 2003; 19:735-43.

20. Goldthorpe JH, Erikson R. The constant flux: a study of class mobility in industrial societies. Oxford: Oxford University Press; 1993.
21. Lopes CS, Faerstein E, Chor D. Eventos de vida produtores de estresse e transtornos mentais comuns: resultados do Estudo Pró-Saúde. Cad Saúde Pública 2003; 19:1713-20.

22. Sknov T, Deddens J, Petersen MR, Endahl L. Prevalence proportion ratios: estimation and hipothesis testing. Internatinal Epidemiology Association 1998; 27:91-5.

23. Cheng Y, Kawachi I, Coakley EH, Scwartz J, Colditz G. Association between psycosocial work characteristics and health functioning in American women: prospective study. BMJ 2000; 320:1432-6.

24. Josephson M, Lagerstrom M, Hagberg M, Wigaeus HE. Musculoskeletal symptoms and job strain among nursing personnel: a study over a three year period. Occup Environ Med 1997; 54:681-5.

25. North FM, Syme L, Feeney A, Shipley M, Marmot, M. Psychosocial work environment and sickness absence among British civil servants: The Whitehall II Study. Am J Public Health 1996; 86:332-40.

26. Demiral Y, Soysal A, Bilgin AC, Killiç B, Unal B, Uçur R, et al. The association of job strain with coronary heart disease and metabolic syndrome in municipal workers in Turkey. J Occup Health 2006; 48:332-8.

27. Ostry AS, Radi S, Louie AM, LaMontagne AD. Psycosocial and others working conditions in relation to body mass index in a representative sample of Australian workers. BMC Public Health 2006; 6:53.

28. Alves, MGM. Pressão no trabalho: Estresse no trabalho e hipertensão arterial em mulheres no Estudo Pró-Saúde [Tese de Doutorado]. Rio de janeiro. Escola Nacional de Saúde Pública, Fundação Oswaldo Cruz; 2004.

Recebido em 15/Mar/2006

Versão final reapresentada em 26/Fev/2007

Aprovado em 16/Abr/2007 